\title{
A neuro-cardiac self-regulation therapy to improve autonomic and neural function after SCl: a randomized controlled trial protocol
}

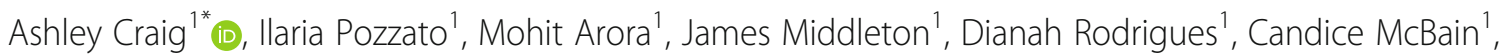
Yvonne Tran², Glen M. Davis ${ }^{3}$, Bamini Gopinath², Annette Kifley ${ }^{1}$, Andrei Krassioukov', Jeffrey Braithwaite ${ }^{5}$, Rebecca Mitchell ${ }^{5}$, Sylvia M. Gustin ${ }^{6}$, Jacob Schoffl' and lan D. Cameron ${ }^{1}$

\begin{abstract}
Background: Spinal cord injury (SCl) is associated with autonomic imbalance and significant secondary conditions, including cardiac and brain dysfunction that adversely impact health and wellbeing. This study will investigate the effectiveness (intention-to-treat) of a neuro-cardiac self-regulation therapy to improve autonomic and neural/brain activity in adults with SCl living in the community.

Methods: A two-arm parallel, randomised controlled trial in which adults with $\mathrm{SCl}$ living in the community postrehabilitation will be randomly assigned to a treatment or control group. The treatment group $(N=60)$ aged 18-70 years with a chronic traumatic or non-traumatic $\mathrm{SCl}$, will receive intervention sessions once per week for 10 weeks, designed to regulate autonomic activity using computer-based feedback of heart rate variability and controlled breathing (called HRV-F). Comprehensive neurophysiological and psychological assessment will occur at baseline, immediate post-treatment, and 6 and 12-months post-treatment. Primary outcome measures include electrocardiography/heart rate variability (to assess autonomic nervous system function) and transcranial doppler sonography (to assess cerebral blood circulation in basal cerebral arteries). Secondary outcomes measures include continuous blood pressure, electroencephalography, functional near-infrared spectroscopy, respiration/breath rate, electrooculography, cognitive capacity, psychological status, pain, fatigue, sleep and quality of life. Controls $(N=60)$ will receive usual community care, reading material and a brief telephone call once per week for 10 weeks and be similarly assessed over the same time period as the HRV-F group. Linear mixed model analysis with repeated measures will determine effectiveness of HRV-F and latent class mixture modelling used to determine trajectories for primary and selected secondary outcomes of interest.
\end{abstract}

\footnotetext{
* Correspondence: a.craig@sydney.edu.au

'John Walsh Centre Rehabilitation Research, Northern Sydney Local Health District, The Kolling Institute, Northern Clinical School, Faculty of Medicine and Health, The University of Sydney, St Leonards, NSW 2065, Australia

Full list of author information is available at the end of the article
}

(c) The Author(s). 2021 Open Access This article is licensed under a Creative Commons Attribution 4.0 International License, which permits use, sharing, adaptation, distribution and reproduction in any medium or format, as long as you give appropriate credit to the original author(s) and the source, provide a link to the Creative Commons licence, and indicate if changes were made. The images or other third party material in this article are included in the article's Creative Commons licence, unless indicated otherwise in a credit line to the material. If material is not included in the article's Creative Commons licence and your intended use is not permitted by statutory regulation or exceeds the permitted use, you will need to obtain permission directly from the copyright holder. To view a copy of this licence, visit http://creativecommons.org/licenses/by/4.0/ The Creative Commons Public Domain Dedication waiver (http://creativecommons.org/publicdomain/zero/1.0/) applies to the data made available in this article, unless otherwise stated in a credit line to the data. 
Discussion: Treatments for improving autonomic function after $\mathrm{SCl}$ are limited. It is therefore important to establish whether a neuro-cardiac self-regulation therapy can result in improved autonomic functioning post-SCl, as well as whether HRV-F is associated with better outcomes for secondary conditions such as cardiovascular health, cognitive capacity and mental health.

Trial registration: The study has been prospectively registered with the Australian and New Zealand Clinical Trial Registry (ACTRN12621000870853.aspx).

Date of Registration: 6th July 2021.

Trial Sponsor: The University of Sydney, NSW 2006.

Protocol version: 22/07/2021.

Keywords: Respiration, Electrocardiography, Electroencephalography, Near infra-red spectroscopy, heart rate variability feedback, cognitive impairment, mood disorder, autonomic nervous system, spinal cord injury.

\section{Background}

Spinal cord injury (SCI) is associated with damage to the nervous system and spinal cord as a result of bruising, compression or severance following a traumatic injury [1], or with deterioration of the spine, infection, vascular accident, or tumours in non-traumatic disease [2]. People with SCI also have higher risks of secondary health conditions, including sleep disorder $[3,4]$, psychological distress [5, 6], chronic pain [6,7], elevated fatigue [8], cognitive impairment $[9,10]$ cardiovascular morbidity $[1,11]$ and autonomic nervous system dysfunction [1, 4, 11]. Dysfunctional autonomic activity after SCI impacts various bodily functions, including cardiac and brain function. However, treatment options and research targeting autonomic dysfunction after SCI are still limited.

Recently, there has been focus on restoring autonomic function in people with SCI using transcutaneous or surgically implanted epidural stimulation technology of the thoracolumbar cord [12, 13]. Preliminary studies have shown favourable results, such as temporary improvements in cardiovascular and motor function [12, 13]. However, there has been scarcely any research investigating benefits of non-invasive neuro-cardiac selfregulation interventions that could enhance neuroplasticity and improve autonomic/neural function following a SCI. Neuro-cardiac self-regulation (HRV-F) usually involves participants learning to regulate their autonomic neuro-cardiac activity via computer/mobile app-based feedback of heart rate variability (HRV) combined with instruction on slow/diaphragmatic breathing, with sessions delivered once per week for between 6 and 10 weeks [14]. Research has shown such neuro-cardiac selfregulation therapy to be a promising treatment for anxiety, depressive mood and trauma-related distress [15], asthma [16], pain [17], and heart disease [18].

The presumed mechanism for benefits associated with self-regulation of the autonomic nervous system involves a combination of physiological processes resulting in strengthening of baroreflex homeostasis, vagal afferent pathways, and phase relationships between blood pressure, heart-rate and brain connectivity [19-22]. Optimal autonomic regulation is associated with healthy functioning of the body's major systems, particularly cardiac and brain function (e.g., cognitive and psychological aspects) [11, 19-22]. Following a SCI, serious cardiovascular complications are a major concern, such as unstable blood pressure control related to episodes of orthostatic hypotension and autonomic dysreflexia (AD), characterized by critical blood pressure rises and associated bradycardia [23-25]. Benefits of improved autonomic self-regulation could therefore be substantial for people with SCI, including reduced cardiovascular morbidity associated with autonomic aetiology, orthostatic instability, severity of $\mathrm{AD}$, and improved cognitive functioning, mental health, pain, vitality and sleep $[4,11-13]$. Furthermore, this study may lead to improved understanding of the physiological underpinning of brainautonomic dysfunction and the influence of top down/ bottom up controls related to neuroplasticity [22].

Evidence that autonomic activity changes after a SCI and during autonomic challenges suggests autonomic modulation may be a new frontier for improving neurocardiac function in individuals with SCI and may mitigate maladaptive plasticity [11]. Underlying hypotheses are the development of intrinsic spinal rhythmicity within the injured cord or recovery of supraspinal pathways [22], demonstrating that autonomic activity measured by HRV in adults with SCI is imbalanced, very different to able-bodied individuals [26]. For example, groups with tetraplegia and paraplegia both have significantly reduced low frequency (LF) HRV activity and impaired sympatho-vagal control [26] and this autonomic imbalance was associated with sleep disorder and fatigue in SCI $[4,26,27]$. It has also been shown that after participating in a 40-min resting/cognitive task, adults with high lesions (at/above T3 level) significantly increased the standard deviation of the average normal-to-normal 
intervals (SDNN) in the HRV in the LF band (0.04-0.15 $\mathrm{Hz}$ ) in comparison to an able-bodied cohort [4], suggesting that low frequency autonomic activity in this group is modifiable.

Dysfunctional brain/neural activity is also reported after SCI, in terms of altered brain activity and connectivity [28-32], cerebral blood circulation [33] and cognitive impairment $[9,10]$. Research has shown that brain activity is related to HRV activity, clarifying mechanisms of heart-brain neurovisceral relationships [28, 29]. For example, in non-SCI samples, HRV biofeedback improved cognitive and brain activity [29] while increases in the $8-12 \mathrm{~Hz}$ electroencephalography (EEG) range (called alpha activity) resulted in changes in HRV [28]. Additionally, adults with SCI have increased risk of thalamocortical dysrhythmia, that is, brain activity characterized by low frequencies (theta/low alpha) similar to aged brain activity in frailty [30, 31]. Furthermore, it is important to improve regulation of cerebral blood flow to compensate for the life-threatening variations in the movement of blood through the circulatory system associated with SCI [33]. It is therefore feasible that HRV self-regulation therapy could result in improved cerebral blood flow, EEG activity, brain metabolism and cognitive capacity in adults with SCI.

The primary objective of this research is to establish the effectiveness of a self-regulation intervention (HRVF) to improve autonomic/neuro-cardiac function in adults with SCI, that is, improved autonomic regulation and brain/neural activity, such as cerebrovascular circulation and brain connectivity. Secondary objectives involve determining whether HRV-F results in improvements in associated functions such as mental health, cognitive capacity, vitality/fatigue, pain and sleep. While there is limited evidence that HRV-F will achieve these objectives in adults with SCI [12,21], the scientific basis for our objectives is compelling. This includes: (i) the fact that a healthy autonomic nervous system is of vital importance for daily functioning given it regulates the respiratory, cardiovascular, digestive, endocrine and neural systems [32]; (ii) we have demonstrated functional/adaptive changes in autonomic activity in adults with chronic SCI (i.e., increased HRV) [4]; and (iii) systematic review and meta-analytic evidence concluded HRV-F is an effective self-regulatory intervention for conditions such as depression, heart disease and other clinical conditions in non-SCI populations $[15,18,34$, 35]. This research will address evidence gaps about improving autonomic/neural function in people with SCI, especially in higher and complete lesions. It will also develop a strategic plan for translating findings to those individuals with SCI living in the community with disability and at-risk from secondary conditions like orthostatic instability and AD.

\section{Methods}

\section{Study design}

The design is a two-arm parallel, intention-to-treat, prospective single-blind randomized controlled trial (RCT).

\section{Objectives \\ Primary objectives}

To establish the effectiveness of a self-regulation intervention (HRV-F) to improve autonomic/neuro-cardiac function in adults with SCI. Autonomic/neuro-cardiac function includes autonomic regulation and brain/neural activity, such as cerebral vascular perfusion and brain connectivity.

\section{Secondary objectives}

1. To determine whether HRV-F results in improvements in associated secondary conditions, such as mental health, cognitive functioning, fatigue, pain, and sleep.

2. To conduct a translation implementation study composed of interviews and a readiness to change survey with a broad range of stakeholders.

\section{Participants}

Participants in the RCT will include 120 adults with a chronic SCI living in the community. Inclusion criteria include: (i) aged 18-70 years with SCI living in New South Wales (NSW), Australia; (ii) English speaking; (iii) traumatic/non-traumatic aetiology with complete/incomplete lesions; (iv) at least 12-months post-injury. Exclusion criteria consist of: (i) evidence of severe cognitive impairment as determined by a neurocognitive screen; (ii) evidence of severe respiratory disorder or impaired respiratory function; (iii) evidence of severe psychiatric disorder, such as bipolar disorder or psychoses, determined by psychiatric assessment, and (iv) taking prescribed ß-blockers. Participants for the implementation study will include up to 100 stakeholders and health professional participants. Stakeholders include individuals from relevant government departments and SCI advocacy groups. Health professionals will include those involved in rehabilitation in the hospital and community settings. Participant recruitment for the implementation study will involve convenience sampling through the NSW Agency for Clinical Innovation State SCI Service (SSCIS) Network and personal contacts in relevant health settings.

\section{Procedure}

The RCT will involve the 120 adults being randomly allocated to either a HRV-F group or a no-treatment control group. Both groups will be similarly assessed over a period of 12-months, that is, at baseline, 10 weeks post- 
baseline (immediately after the HRV-F treatment for the intervention group), and at 6 and 12-month postbaseline. To test the feasibility of the RCT, the first 10 RCT participants with SCI who meet inclusion and exclusion criteria (5 in the HRV-F and 5 in the control arm) will be monitored for adherence to the HRV-F therapy and assessment, and for any barriers to the intervention and control group membership. The implementation study will involve conducting a mixed methods approach (qualitative and quantitative) to establish how best to translate HRV-F interventions into the community.

\section{Data management}

After recruitment, participants will be de-identified and given a generated code. All data will be stored on a secure online platform called Research Electronic Data Capture (REDCap) [36]. Participants will enter their responses to the online interview/assessment using their personal REDCap link after being given instructions on how to complete. Participants not wanting to enter responses online will complete by face-to-face or telephone assessment with a member of the research team, who will then enter the data onto REDCap. The implementation phase participants will also be de-identified and data from the surveys and interviews will be entered into REDCap for further analysis.

\section{Randomization and blinding}

RCT participants will be randomized to either the HRVF group $(N=60)$ or to the no-treatment control group $(\mathrm{N}=60)$ following a stratified randomization procedure. The randomisation allocation ratio is $1: 1$, while controlling for covariate level of injury (at and above T3 versus below T3). The randomization sequence will be computer generated via RAND function in Microsoft Excel by an independent person and then be uploaded into REDCap. Once the participant is recruited and completed the baseline assessment, then the assessor will inform the team members who are conducting the intervention. The researcher will then randomize a participant via pre-defined randomization sequence in REDCap (see Fig. 1). Team members responsible for recruitment, assessment and analysing the data will be blinded to group allocation. However, the researcher conducting the intervention as well as the participants will be aware of group allocation given it is not possible to blind them to the HRV-F versus control conditions.

\section{Setting}

Participants living in the community will be recruited from outpatient clinics of the two participating sites, that is, Royal North Shore Hospital (RNSH) and Royal Rehab, NSW, or they will approach the research team after viewing study advertisements posted at targeted social and commercial media outlets. Assessment and the

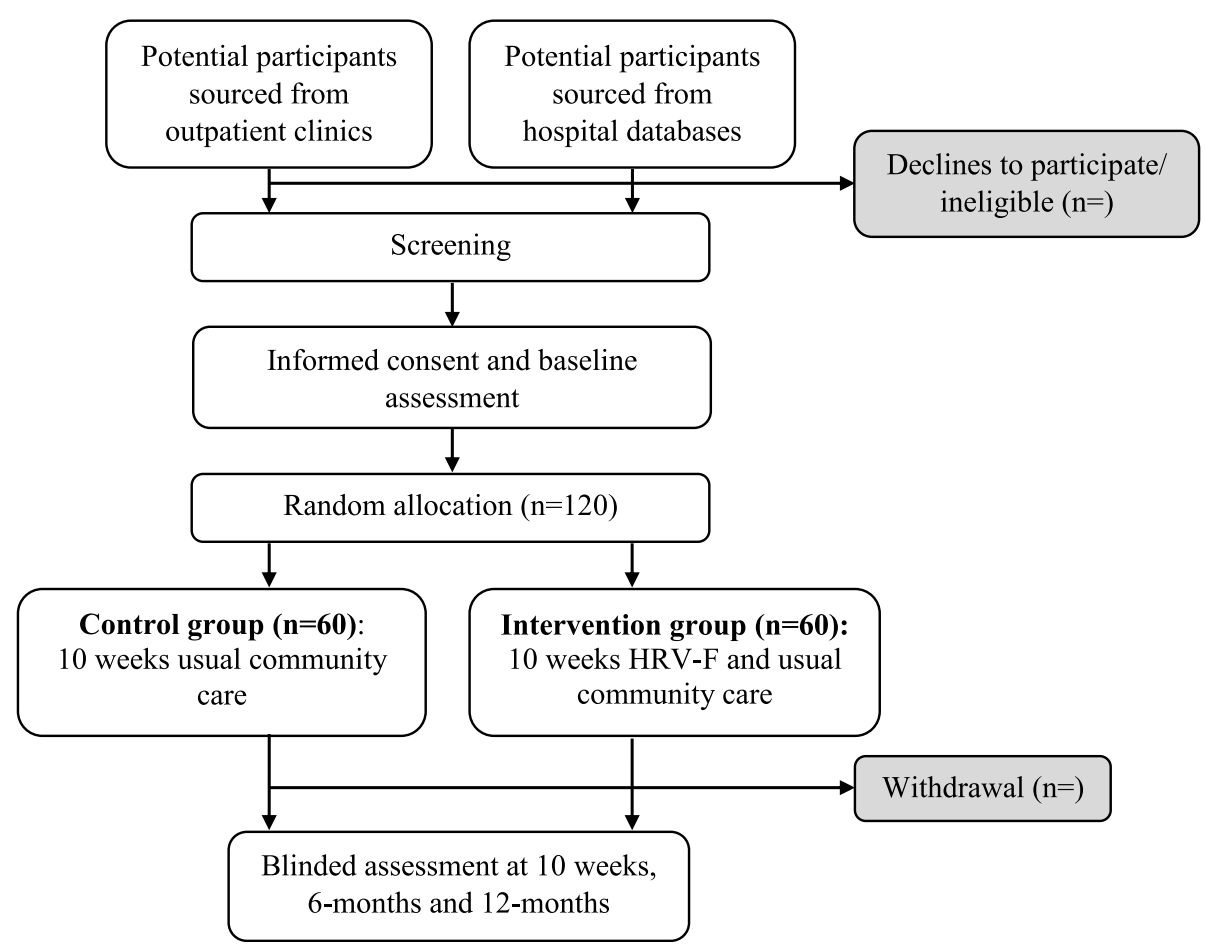

Fig. 1 Showing participant allocation to the HRV-F versus Control conditions 
HRV-F intervention will only be conducted at the RNSH campus in which the testing laboratory, a dedicated airconditioned laboratory, is situated. Participants in the HRV-F group will also complete some treatment components in their home setting. The implementation phase participants from the SSCIS Network will be asked to complete an online survey. The SSCIS Network members include clinicians, consumers, government policymakers and representatives from non-government organisations. Ten semi-structured interviews will be conducted via telephone with health professionals who have delivered the HRV-F intervention, or who would deliver the HRV-F if it was introduced into standard practice.

\section{HRV-F protocol and rationale Rationale}

HRV-F is designed to improve autonomic and neurocardiac functioning using computer-based feedback of HRV and controlled diaphragmatic slow breathing around 37 breaths per minute. One goal of HRV biofeedback is to stimulate the baroreflex and enhance respiratory sinus arrhythmia (RSA), which involves fluctuations in heartbeat intervals linked with respiration, associated with variations of the parasympathetic cardiac signal [37]. That is, cognition and brain neural activity to adhere to a paced respiration rate and depth, evoking resonant frequency changes to RSA in LF HRV and Total HRV power (in the spectral bands). RSA amplitude (peak-totrough heart rate difference across the breathing cycle) is contingent with slow breathing [37] and establishing resonant breathing frequency (generally lying between 4 and 7 breaths per minute), identifies the breath rate that is associated with largest gains in RSA and baroreflex activity [37]. Slow breathing rates, and especially breathing at the resonant breath rate, optimally stimulates the baroreflex, resulting in increased RSA and HRV, which is believed to be associated with healthy autonomic and cardiovascular systems [37].

\section{Resonant frequency}

A central goal of HRV-F, therefore, is to establish a participant's resonant breath rate [14, 19, 37]. Participants utilize feedback of their HRV in exercises to control their autonomic activity using dedicated biofeedback technology and software (ProComp 2 HRV Systems; Thought Technology, Ltd., Canada, www. thoughttechnology.com). In HRV-F, participants initially use this feedback technology to determine their unique resonant breathing rate, which is established over a period of $15 \mathrm{~min}$ while reducing their breath rate by $0.5-1$ breaths per minute (BPM), and depending on a participant's initial resting BPM, gradually decreasing $\mathrm{BPM}$ down to 4 or $5 \mathrm{BPM}$ over the $15-\mathrm{min}$. Once participants have identified their unique resonant breathing frequency, they then proceed to perform varied resonant breathing exercises over the 10-week period. The protocol is shown in Fig. 2.

\section{HRV-F training}

Ten sessions of formal HRV-F training will be completed over the 10-week intervention. These will involve a mixture of six face-to-face formal sessions (2-h duration each) in the research laboratory and four online/ telephone sessions (at-home sessions, up to 1-h duration). Face-to-face HRV-F sessions will be conducted in groups of three participants. However, it is expected that individual sessions will occur given risk of sickness in this population. Self-management strategies will be introduced throughout the 10 weeks of the program, including self-monitoring of mood, anxiety, breath rate, pain/fatigue level and sleep quality, as well as visualisation strategies to be used in conjunction with resonant breathing exercises.

\section{Home practice}

Homework related to HRV-F will be assigned and a diary used by participants to log homework activities. Homework activities will include (i) resonant breathing exercises as taught in the face-to face sessions; (ii) measuring HRV each day of the 10 weeks using phone-based apps; (iii) self-monitoring of mood, anxiety, breath/heart rate, pain, fatigue and sleep; (iv) maintaining a $\log$ of homework activities, and (v) visualisation strategies to enhance their self-regulation of HRV and breathing. Participants will be asked to practise resonant breathing exercises in the home environment, twice a day for around 20 min per session and once a week over the 10 weeks, a team member will provide telephone support. Phone apps will be used to record HRV data and determine adherence, as well as daily/weekly progress. All HRV-F participants will be given a total of $\$ 200$ Australian in the form of eGift cards throughout the treatment program ( $\$ 50$ at initial and $\$ 50$ at 10 weeks) and a final $\$ 100$ after the 12-month assessment.

\section{Control group protocol}

Control participants will receive the same assessment regime as the HRV-F participants. Controls will receive reading material and a brief telephone call each week in the 10-week period to establish their continued participation. Controls will receive similar payment to the intervention group.

\section{Implementation phase}

Guided by the Theoretical Domains Framework (TDF) [38], the implementation phase participants will be asked to complete an online survey using REDCap to identify 


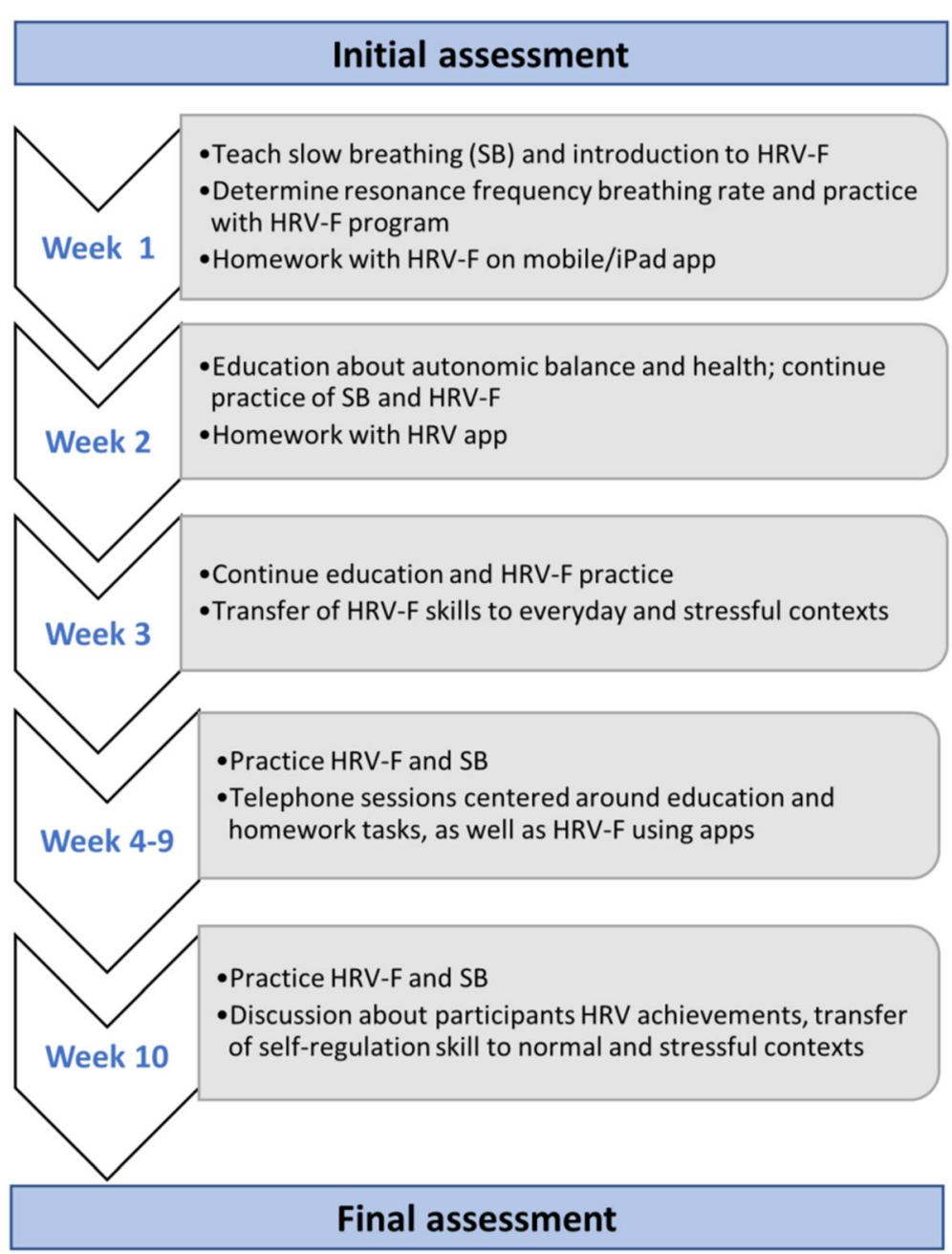

Fig. 2 Shows flow of HRV-F intervention sessions over 10 weeks

facilitators and barriers to the introduction of HRV-F into standard practice. Survey responses will be used to establish how best to translate the HRV-F intervention into healthcare practice for adults with SCI. Survey participation will be voluntary and anonymous. Semistructured interviews will be conducted via telephone with health professionals who have delivered the HRV-F intervention, or who would deliver the HRV-F, if it was introduced into standard practice. This will include professionals such as psychologists, physiotherapists, nurses, rehabilitation physicians, social workers and occupational therapists. The semi-structured interviews will be used to reflect on the facilitators and barriers identified during the survey and to review the practicality and acceptability of potential solutions to identified barriers. The participants who completed the HRV-F will also be asked two open-ended questions after they complete HRV-F:
"What have been the main challenges for you with learning and doing your therapy?" and "If there was one or two things that could be done differently to improve your therapy, what would they be?" The implementation at scale model to support systems-level diffusion (see Fig. 3), will also inform this work [39]. Articulating a robust implementation strategy addressing challenges faced by health professionals and stakeholders will streamline future translation, preventing costly trial and error attempts in implementing HRV-F into practice.

\section{Socio-demographic, injury and medical assessments}

Socio-demographic assessment includes sex, age at the time of initial measurement, level of education and employment history. Injury details include age at the time of the SCI, time (years) since the SCI, level and extent (completeness) of the injury using the International Standards for Neurological Classification of SCI (ISNC 


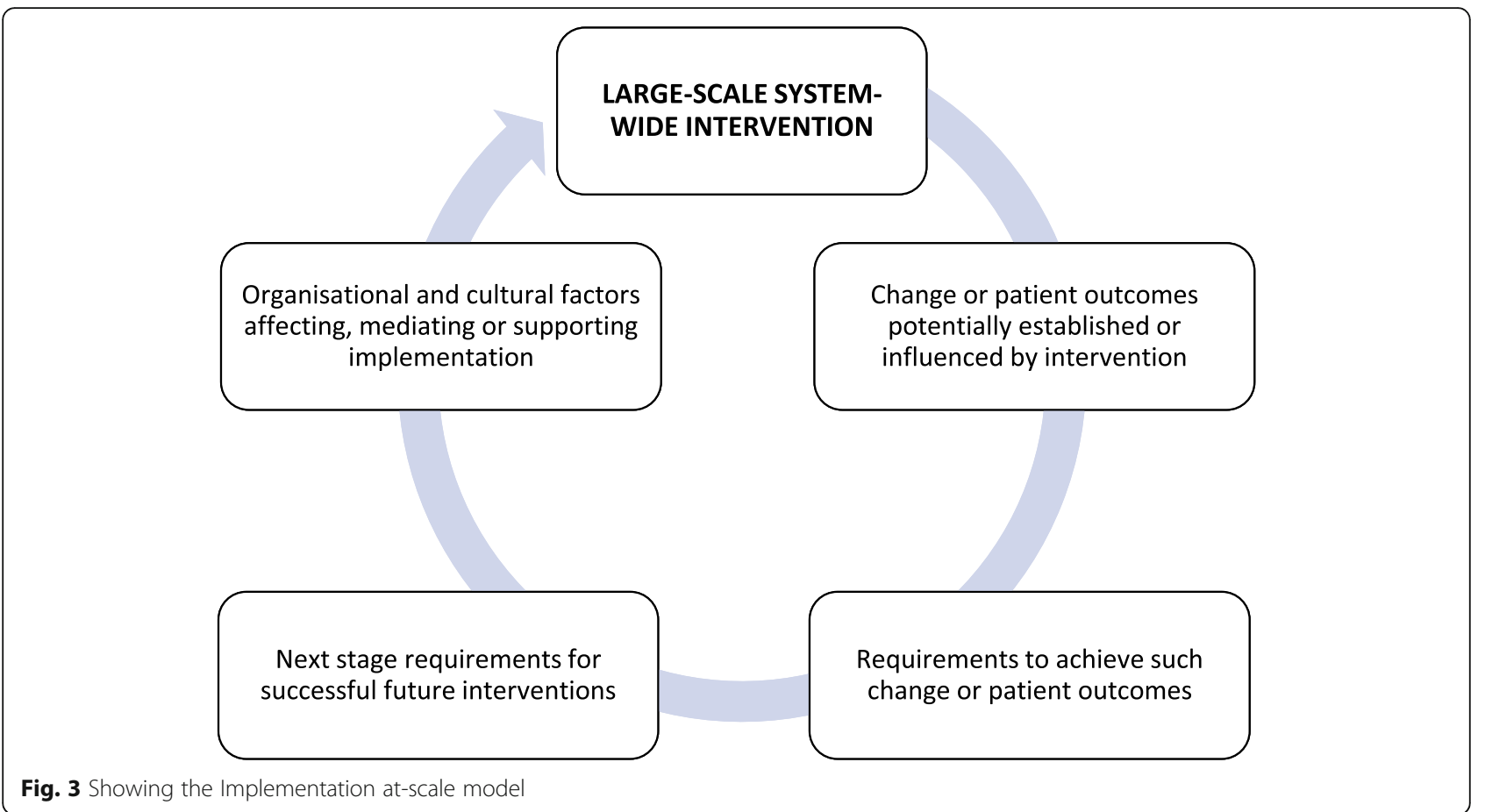

SCI; https://asia-spinalinjury.org/isncsci-2019-revision). Medical assessment includes medical history (e.g., pre and post-injury health and psychological status), current medications, body mass index assessed from selfreported height and weight, and classified according to World Health Organization guidelines: $<18.5 \mathrm{~kg} / \mathrm{m}^{2}$ (underweight), $18.5-24.9 \mathrm{~kg} / \mathrm{m}^{2}$ (normal), $25-29.9 \mathrm{~kg} / \mathrm{m}^{2}$ (overweight), $\geq 30 \mathrm{~kg} / \mathrm{m}^{2}$ (obese).

\section{Physiological assessment}

See Table 1 for all primary and secondary outcome measures and endpoints (baseline versus 10 weeks, 6 and 12 months) for the RCT and implementation phase. The physiological assessment protocol includes:

(i) Activity \#1: 5-min resting baseline (spontaneous breathing) after 2-min habituation;

(ii) Activity \#2: 5-min Stroop test;

(iii) Activity \#3: 5-min recovery (spontaneous breathing);

(iv) Activity \#4: 5-min breathing task (paced breathing at around 6 breath/minute);

(v) Activity \#5: 5-min recovery (spontaneous breathing).

\section{Primary physiological outcome measures}

- Electrocardiography (ECG) assessment from which HRV will be calculated at 10 weeks (short-term effect), in order to determine whether HRV-F results in improved autonomic nervous system function. HRV via ECG (electrodes on chest, modified lead II configuration) will be measured using the PowerLab 8/35 8-channel, 16-bit resolution recorder data acquisition system with programmable gain and two galvanically-isolated channels FE232 Dual Bio Amps (ADInstruments, www.adinstruments.com). A sampling rate of $512 \mathrm{~Hz}$ will be used for time/spectral analyses [4]. HRV signal processing will be performed using Kubios HRV Premium software (Version 3.3.1, University of Kuopio, Finland). HRV indexes neuro-cardiac function/regulation, generated by bidirectional heart-brain interactions (i.e., topdown influences on cardiac and peripheral physiological processes and bottom-up influences on central control mechanisms) and dynamic non-linear autonomic nervous system (ANS) regulation. HRV reflects the innervation of the heart by the sympathetic/parasympathetic divisions of the ANS [4]. In the time domain, average and standard deviation of the normal-to-normal intervals (SDNN; the variability of the heart) and root mean square of the successive differences (RMSSD, vagally mediated HRV) will be calculated. In the frequency domain, low frequency (LF), high frequency (HF, cardiac vagal 
Table 1 Schedule of activities and measures for the RCT and implementation study. All psychometric instruments are scientifically validated and reliable

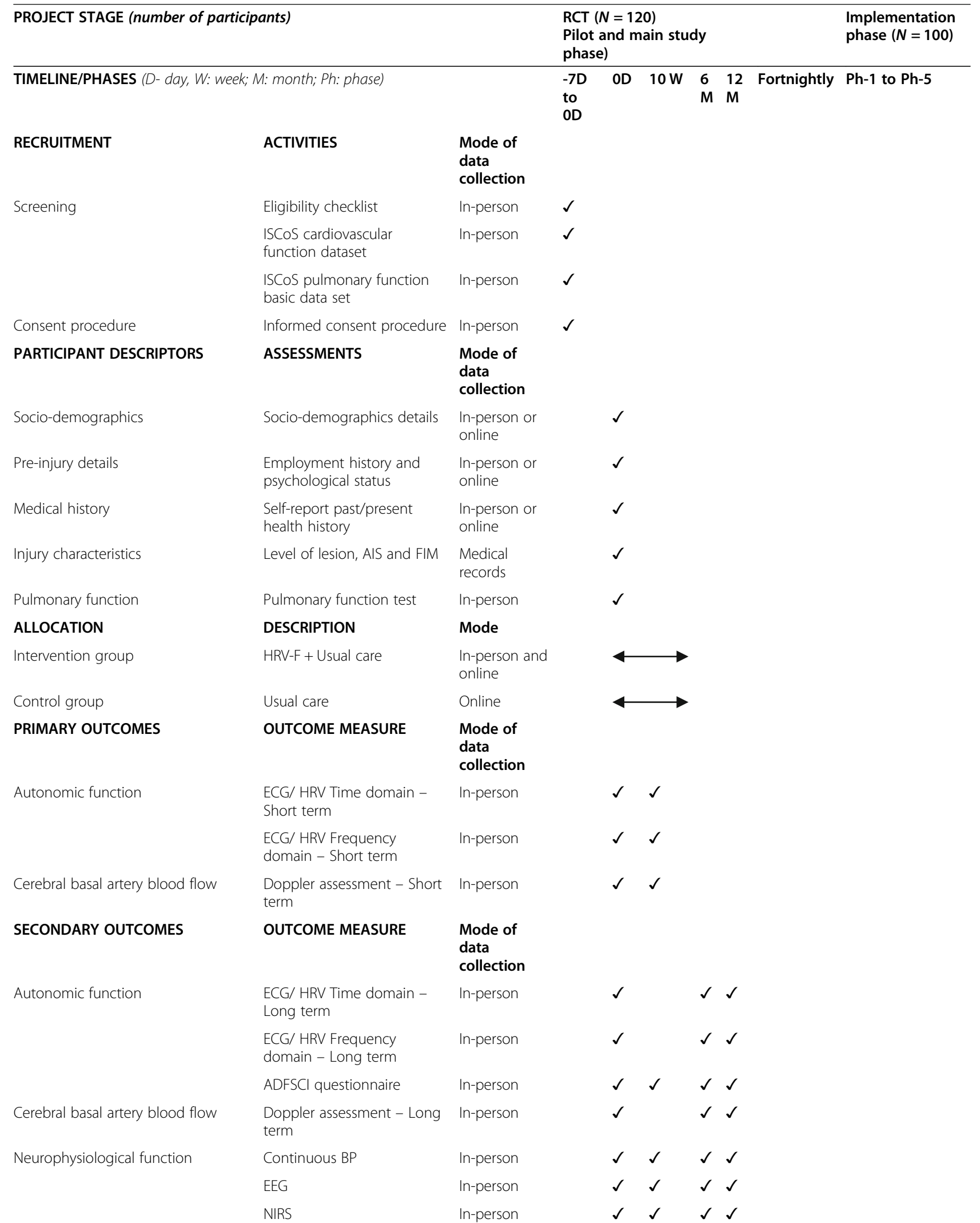


Table 1 Schedule of activities and measures for the RCT and implementation study. All psychometric instruments are scientifically validated and reliable (Continued)

\begin{tabular}{|c|c|c|c|c|c|c|c|}
\hline \multicolumn{3}{|c|}{ PROJECT STAGE (number of participants) } & \multicolumn{4}{|c|}{$\begin{array}{l}\text { RCT }(N=120) \\
\text { Pilot and main study } \\
\text { phase) }\end{array}$} & \multirow[t]{2}{*}{$\begin{array}{l}\text { Implementation } \\
\text { phase }(N=100)\end{array}$} \\
\hline & EOG & In-person & $\checkmark$ & $\checkmark$ & $\checkmark$ & $\checkmark$ & \\
\hline & $\begin{array}{l}\text { Skin conductance, BVP, } \\
\text { respiration and temp }\end{array}$ & In-person & $\checkmark$ & $\checkmark$ & $\checkmark$ & $\checkmark$ & \\
\hline Secondary health conditions & SCI-SCS & $\begin{array}{l}\text { In-person and } \\
\text { online }\end{array}$ & $\checkmark$ & $\checkmark$ & $\checkmark$ & $\checkmark$ & \\
\hline \multirow[t]{2}{*}{ Cognitive function } & NUCOG & $\begin{array}{l}\text { In-person and } \\
\text { online }\end{array}$ & $\checkmark$ & $\checkmark$ & & & \\
\hline & Stroop test & $\begin{array}{l}\text { In-person and } \\
\text { online }\end{array}$ & $\checkmark$ & $\checkmark$ & & & \\
\hline \multirow[t]{2}{*}{ Psychological/mental health function } & GADS & $\begin{array}{l}\text { In-person and } \\
\text { online }\end{array}$ & $\checkmark$ & $\checkmark$ & $\checkmark$ & $\checkmark$ & \\
\hline & PHQ-9 & $\begin{array}{l}\text { In-person and } \\
\text { online }\end{array}$ & $\checkmark$ & $\checkmark$ & $\checkmark$ & $\checkmark$ & \\
\hline \multirow[t]{4}{*}{ Psychosocial function } & ISCoS pain questionnaire & $\begin{array}{l}\text { In-person and } \\
\text { online }\end{array}$ & $\checkmark$ & $\checkmark$ & $\checkmark$ & $\checkmark$ & \\
\hline & FSS & $\begin{array}{l}\text { In-person and } \\
\text { online }\end{array}$ & $\checkmark$ & $\checkmark$ & $\checkmark$ & $\checkmark$ & \\
\hline & PSQA & $\begin{array}{l}\text { In-person and } \\
\text { online }\end{array}$ & $\checkmark$ & $\checkmark$ & $\checkmark$ & $\checkmark$ & \\
\hline & PCS & $\begin{array}{l}\text { In-person and } \\
\text { online }\end{array}$ & $\checkmark$ & $\checkmark$ & $\checkmark$ & $\checkmark$ & \\
\hline Personal factors & CD-RISC2 Short Form & $\begin{array}{l}\text { In-person and } \\
\text { online }\end{array}$ & $\checkmark$ & $\checkmark$ & $\checkmark$ & $\checkmark$ & \\
\hline Quality of life & $E Q-5 D-5 L$ & $\begin{array}{l}\text { In-person and } \\
\text { online }\end{array}$ & $\checkmark$ & $\checkmark$ & $\checkmark$ & $\checkmark$ & \\
\hline Participation and disability & $\begin{array}{l}\text { WHODAS domain - } \\
\text { participation items }\end{array}$ & $\begin{array}{l}\text { In-person and } \\
\text { online }\end{array}$ & $\checkmark$ & $\checkmark$ & $\checkmark$ & $\checkmark$ & \\
\hline $\begin{array}{l}\text { Consumer perception and } \\
\text { satisfaction }\end{array}$ & $\begin{array}{l}\text { Self-reported perception of } \\
\text { change and satisfaction }\end{array}$ & $\begin{array}{l}\text { In-person and } \\
\text { online }\end{array}$ & & $\checkmark$ & & & \\
\hline Costs for Economic evaluation & $\begin{array}{l}\text { Self-reported direct and } \\
\text { indirect costs }\end{array}$ & $\begin{array}{l}\text { Diaries (up to } \\
12 \mathrm{~m} \text { ) }\end{array}$ & & & & $\checkmark$ & \\
\hline Adverse events & $\begin{array}{l}\text { Self-monitoring of Adverse } \\
\text { events }\end{array}$ & $\begin{array}{l}\text { Diaries (up to } \\
12 \mathrm{~m} \text { ) }\end{array}$ & & & & $\checkmark$ & \\
\hline $\begin{array}{l}\text { Self-monitoring of mood, breath rate, } \\
\text { pain, fatigue and sleep quality }\end{array}$ & Numeric rating scale (0 to 10) & $\begin{array}{l}\text { Diaries (up to } \\
12 \mathrm{~m} \text { ) }\end{array}$ & & & & $\checkmark$ & \\
\hline EXPLORATORY OUTCOMES & OUTCOME MEASURE & $\begin{array}{l}\text { Mode of } \\
\text { data } \\
\text { collection }\end{array}$ & & & & & \\
\hline \multirow[t]{4}{*}{ Personal factors } & WHO ASSIST & $\begin{array}{l}\text { In-person and } \\
\text { online }\end{array}$ & $\checkmark$ & $\checkmark$ & $\checkmark$ & $\checkmark$ & \\
\hline & COPE inventory - brief & $\begin{array}{l}\text { In-person and } \\
\text { online }\end{array}$ & $\checkmark$ & $\checkmark$ & $\checkmark$ & $\checkmark$ & \\
\hline & ADAPSS - Short-form & $\begin{array}{l}\text { In-person and } \\
\text { online }\end{array}$ & $\checkmark$ & $\checkmark$ & $\checkmark$ & $\checkmark$ & \\
\hline & MSES & $\begin{array}{l}\text { In-person and } \\
\text { online }\end{array}$ & $\checkmark$ & $\checkmark$ & $\checkmark$ & $\checkmark$ & \\
\hline Psychological/mental health function & PTSD Checklist 5 & $\begin{array}{l}\text { In-person and } \\
\text { online }\end{array}$ & $\checkmark$ & $\checkmark$ & $\checkmark$ & $\checkmark$ & \\
\hline \multirow[t]{2}{*}{ Psychosocial function } & SSQ6 & $\begin{array}{l}\text { In-person and } \\
\text { online }\end{array}$ & $\checkmark$ & $\checkmark$ & $\checkmark$ & $\checkmark$ & \\
\hline & Berlin Questionnaire & In-person and & $\checkmark$ & $\checkmark$ & $\checkmark$ & $\checkmark$ & \\
\hline
\end{tabular}


Table 1 Schedule of activities and measures for the RCT and implementation study. All psychometric instruments are scientifically validated and reliable (Continued)

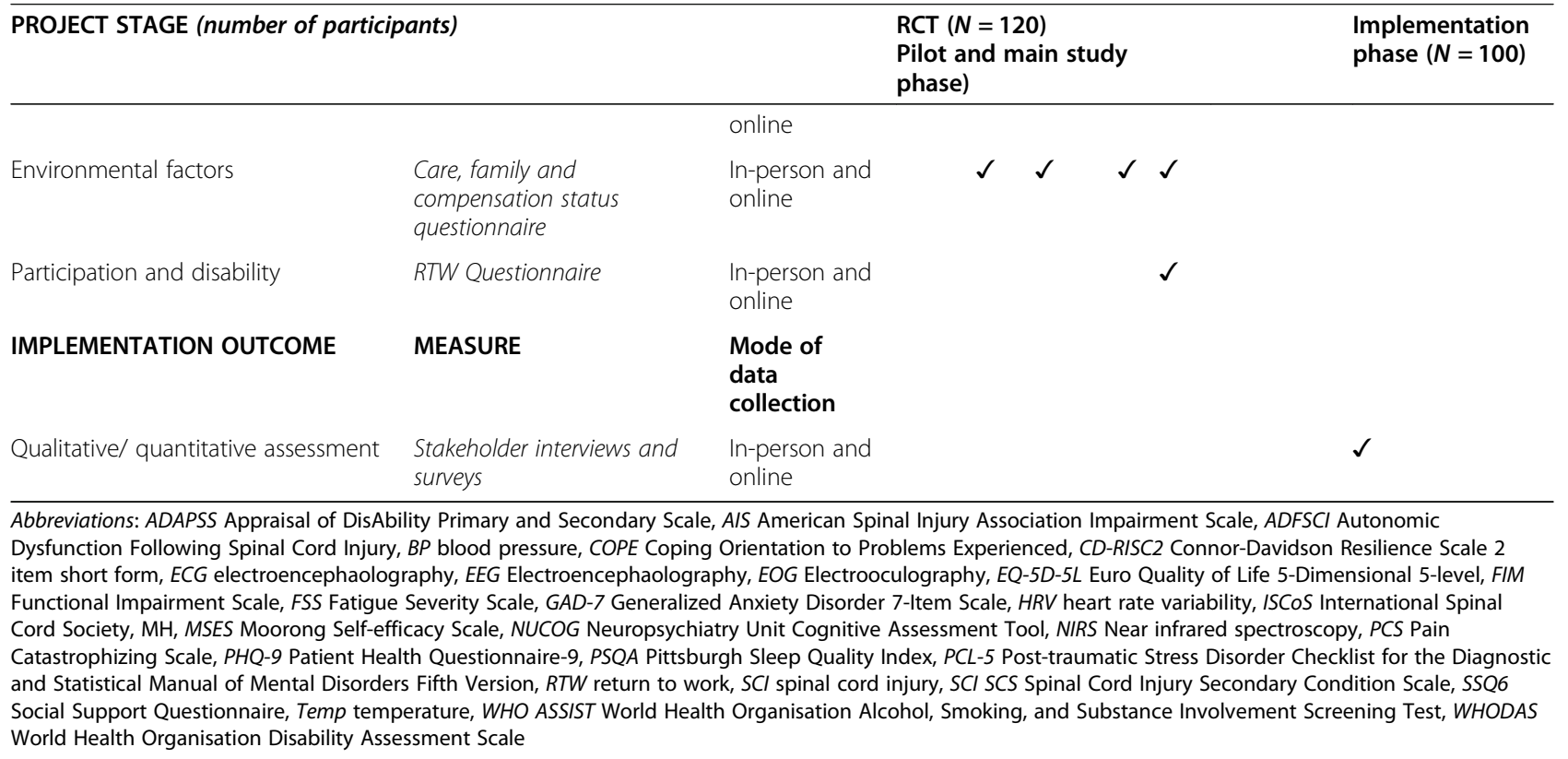

control) and total power will be assessed. Details of a HRV protocol we have employed are available [40].

(ii) Functional transcranial doppler sonography (fTCD) to assess cerebral artery blood flow at 10 weeks (short-term effect), to determine cerebrovascular benefits associated with the HRV-F, using MultiDop T digital with QL routine software (Compumedics Limited, www.compumedics.com.au). fTCD assesses real-time cerebral blood flow velocities/ circulation in basal cerebral arteries of the participants in response to the intervention [41]. fTCD is a reliable method for measuring cerebrovascular changes, provides high temporal resolution and is least susceptible to artefact than other imaging techniques [41].

Electrocardiography (ECG) assessment and Functional transcranial doppler sonography will be performed using the same instruments (as per primary outcomes) at 6 and 12 months (long-term effects).

\section{Secondary physiological outcome measures} Electroencephalography (EEG), electrooculography (EOG), respiration/breath rate, skin conductance, blood volume pulse and finger temperature

To measure these secondary outcome measures, two FlexComp electrophysiological monitoring systems (each having 10-channels each; Thought Technology, Ltd., Canada, www.thoughttechnology.com) with BioGraph Infiniti software, will be used for data acquisition of EEG (11 channels using International 10-20 montage system: F3, Fz, F4, C3, Cz, C4, P3, Pz, P4, O1, O2), skin conductance ( 2 channels, right and left hand), blood volume pulse ( 2 channels, right and left hand), EOG (1 channel), respiration (1 channel) and peripheral skin temperature ( 2 channels, right and left hand). The FlexComp has a sampling rate of 2048 samples/sec with 14 bits of resolution (1 part in 16,364). EEG will be used to assess change in brain wave activity in response to the HRV-F and will be spectrally analysed using fast Fourier Transform and a Hanning Window. Sophisticated noise/artefact removal strategies will be used to improve noise: signal ratio [31]. EOG will be used as a physiological measure of fatigue [40]. Skin conductance, blood volume pulse and finger temperature will assess sympathetic arousal and respiration breath rate [42].

\section{Near infrared spectroscopy}

The Brite MKII 27-channel functional near infrared spectroscopy (fNIRS) system (Artinis Medical Instruments B.V., Amsterdam, Netherlands) will be used to quantify brain oxygenation and neurological activation associated with the HRV-F intervention and complement the EEG measures. NIRS data analysis will be via the OxySoft (Artinis Medical Instruments B.V., Amsterdam, Netherlands) dedicated software used to collect, store, view, and analyse all NIRS signals [43]. 


\section{Continuous blood pressure}

Human NIBP Nano Foundation System (ADInstruments, www.adinstruments.com) will be used to record continuous blood pressure (BP) via non-invasive dual finger cuff system (Labchart software). This will ensure measurements of systolic and diastolic BP as well as BP variability (BPV), to quantify cardiovascular benefits associated with the HRV-F. Time series and spectral analysis of BPV and HRV will be used to evaluate baroreflex function/sensitivity and overall (cardiovascular) autonomic balance, with LF HRV indexing sympathetic activity and HF-HRV indexing cardiac vagal control.

\section{Assessment protocol}

Primary and secondary physiological outcomes will be simultaneously recorded with participants sitting in their wheelchair, according to the following protocol: (i) resting phase of $5 \mathrm{~min}$ (after $2 \mathrm{~min}$ of habituation); (ii) mental challenge task (e.g. Stroop test) of $5 \mathrm{~min}$; (iii) recovery phase of $5 \mathrm{~min}$ (resting and spontaneous breathing); (iv) breathing task of 5 min (paced breathing at 6 breath/min); 5 -min recovery of 5 min (resting and spontaneous breathing). To control circadian influences, assessment will occur in the mornings $(9 \mathrm{am}-1 \mathrm{pm})$ and will be conducted in a quiet temperature-controlled room in the research laboratory. Participants will be asked to refrain from alcohol, caffeine and smoking at least $12 \mathrm{~h}$ before the assessment and to empty their bladder at the arrival to the laboratory.

\section{Secondary outcome measures- self-report (online) interview/assessment}

\section{Autonomic dysfunction Follgeowing spinal cord injury} (ADFSCI)

This questionnaire is a measure of self-reported frequency and severity of symptoms during hypo- and hypertensive episodes [44]. It has been shown to correlate with blood pressure (BP) instability and to have acceptable test-retest reliability [44].

\section{Spinal cord injury secondary conditions scale (SCI-SCS)}

SCI-SCS assesses secondary physiological conditions that are associated with SCI using a 4-point ordinal 16-item scale ranging from 0 (not experienced/insignificant problem) to 3 (significant/chronic problem) [45]. Participants rate how these conditions have affected their activities and independence in the last 3-months. Examples of secondary conditions include pressure ulcers and bladder dysfunction. Higher scores indicate more significant impact [45].

\section{Cognitive assessment}

The Neuropsychiatry Unit Cognitive Assessment Tool (NUCOG) [46, 47], a neurocognitive measure validated for SCI, will be used to assess cognitive functioning using age-adjusted normative scores from a scoring manual. The NUCOG domains include: attention, visuoconstructional ability, memory, executive, and language. Items needing hand function (e.g., object reproduction) will be modified as in prior studies where this has been demonstrated not to alter validity of NUCOG scores [47]. Where it is not possible to administer NUCOG assessments face-to-face (e.g., due to social distancing restrictions due to COVID-19), administration will occur by online methods [10].

\section{Generalised anxiety Disorder-7 (GAD-7)}

GAD-7 is a seven-item psychometric tool that assesses severity of generalized anxiety and probable generalized anxiety disorder (GAD) [48]. Items request individuals to rate severity of anxiety symptoms experienced over the prior 2 weeks. High scores represent more severe generalized anxiety symptoms. GAD-7 has demonstrated reliability and validity [48].

\section{Patient health Questionnaire-9 (PHQ-9)}

PHQ-9 assesses nine Diagnostic and Statistical Manual of Mental Disorders (DSM) criteria for depressive disorder over the prior two-week period [49]. Items range between 0 'not at all' to 3 'nearly every day', with higher scores indicating more severe depressive mood. PHQ-9 has demonstrated reliability and validity [49].

\section{International spinal cord injury basic pain dataset}

This includes four numerical rating items ranging from 0 (no pain) to 10 (extreme or worst pain) for pain intensity and pain interference in day-to-day activities, mood and sleep [50]. Numerical pain rating scales have demonstrated acceptable test-retest reliability and validity [51].

\section{Fatigue severity scale (FSS)}

The FSS is a 9-item self-report scale that measures fatigue and its influence on lifestyle and function [52]. Items are scored on a 7-point Likert scale ( $1=$ strongly disagree and $7=$ strongly agree). Higher scores suggest more severe fatigue. The FSS has demonstrated validity [52].

\section{The Pittsburgh sleep quality index (PSQI)}

PSQI measures sleep quality across seven domains: subjective sleep quality, sleep latency, sleep duration, habitual sleep efficiency, sleep disturbances, use of sleep medication, and daytime dysfunction [53]. Scoring employs a 0-3 Likert scale, with high scores indicating poor sleep. The PSQI has demonstrated validity [53]. 


\section{Pain catastrophizing scale (PCS)}

The PCS is a cognitive bias self-report scale consisting of 13-items that measure degree of catastrophizing type thinking a person is using (e.g., "I can't stop thinking about how much it hurts"). Items are scored 0-4, with a total score of 54 . Higher scores suggest more significant cognitive bias/pain catastrophizing [54]. The PCS has been shown to be reliable and valid [54].

\section{Connor-Davidson resilience scale (CD-RISC2)}

CD-RISC2 is a 2-item brief version of the 25-item CDRISC. It measures perceived resilience over the past month with higher scores reflecting greater resilience [55]. The two items are 'able to adapt to change' and 'tend to bounce back after illness or hardship'. The CDRISC2 has demonstrated internal consistency, test-retest reliability, convergent validity, and divergent validity, and correlates well with the full scale [55].

\section{EUROQOL version $5 D-5 L$ (EQ-5D-5L)}

EQ-5D-5L includes five dimensions about aspects of health, that is, mobility, self-care, usual activities, pain/discomfort, and anxiety/depressive mood [56]. Dimensions have five levels of response, from "no problems" to "extreme problems". Participants are asked to indicate the most appropriate response-level for each of the five dimensions. The EQ-5D-5L has demonstrated validity [56].

\section{World Health Organization disability assessment schedule- participation domain (WHODAS-2)}

The WHODAS-2 is disability assessment instrument based on the International Classification of Functioning, Disability, and Health (ICF) [57]. It provides a global measure of disability and 7 domain-specific scores. Only the Participation domain is being assessed. The WHODAS-2 has demonstrated validity [57].

\section{Self-reported satisfaction with health services received and change in health}

Two in-house Likert scales that assess the participant's perceived satisfaction with received health services and their change in their health over the past 3 months.

Health-related cost, adverse events and monitoring of the mood, breath rate, pain, fatigue and sleep quality

These variables will be measured using a fortnightly participant's diaries.

\section{Exploratory outcome measures- self-report (online) interview/assessment}

Post-traumatic stress disorder checklist short-form (PCL-5-

SF)

PCL-5-SF is a 4-item version of the 20-item PCL-5. This abbreviated version has demonstrated reliability for detecting symptoms of post-traumatic stress disorder (PTSD) [58]. Participants are asked to rate symptoms experienced over the past 4 weeks including avoidance, negative changes in cognition and mood, and changes in arousal and reactivity. This is done on a 5-point Likert scale from 'not at all' to 'extremely often'. Higher scores suggest more severe traumatic stress [58].

\section{Brief coping orientation to problems experienced (brief COPE)}

Brief COPE is a 28-item self-report questionnaire assessing ways of coping with stressful life events [59]. Only questions 2, 3, 7, and 8 of the Brief COPE will be included to minimize assessment time. The four items assess 'Active - Approach' and 'Avoidant - Denial' coping styles [59].

\section{Social support questionnaire short form (SSQ-6)}

The SSQ-6 is a 6-item questionnaire assessing perceived social support, such as the number of people participants believe are available for social support, and how satisfied they are with the level of support they receive. Items are scored from 'very dissatisfied' to 'very satisfied' on a 6-point Likert-type scale. The SSQ6 has demonstrated validity [60].

\section{Moorong self-efficacy scale (MSES)}

MSES measures self-efficacy related to expectations of control about functional activities of daily living in individuals with SCI [61]. Participants are asked to rate perceptions of their belief about their ability to manage 16 tasks on a 7-point Likert scale with 1 being very uncertain and 7 being very certain. Higher scores suggest higher perceived self-efficacy to perform functional activities [61].

\section{Appraisals of DisAbility: primary and secondary scale (ADAPSS) short-form}

ADAPSS Short-Form measures SCI-specific appraisals using 6 items from the full ADAPSS scale. It has a 2factor structure of "catastrophic negativity" and "determined resilience". The ADAPSS Short-form has demonstrated validity [62].

\section{Berlin questionnaire (BQ)}

The Berlin questionnaire is a questionnaire used to assess obstructive sleep apnea. It involves 11 items about snoring, breathing problems during sleep and fatigue during wake hours [63]. The BQ has demonstrated validity and sensitivity/specificity [63]. 


\section{World Health Organisation alcohol, smoking, and substance involvement screening test (WHO ASSIST)}

Question 2 of the WHO ASSIST requests information on the frequency of use of ten substances during the past 3 months, including substances such as tobacco, alcohol, cannabis, cocaine, amphetamine-type stimulants, inhalants, sedatives, hallucinogens, opioids, and 'other' drugs [64]. Responses are scored on a 5-point Likert scale, from 'never' to 'daily or almost daily.' Reliability of the WHO ASSIST has been demonstrated [65].

\section{Return to work questionnaire}

Return to work will be measured using a return to work questionnaire designed especially for the study with 5items related to employment nature and status.

\section{Environmental factor questionnaire}

Care, family and compensation status will be assessed using a questionnaire designed especially for the study with 7-items.

\section{Implementation participant survey and semi-structured interviews}

Participant surveys will include a mix of positive and negatively phrased closed and open-end questions based on the 14 TDF domains designed to identify facilitators and barriers to the introduction of HRV-F. The semistructured interviews will ask participants to reflect on the facilitators and barriers identified during the survey and to comment on the practicality and acceptability of potential solutions to identified implementation barriers.

\section{Economic evaluation}

An economic assessment will be conducted that will include costs potentially incurred by participants and healthcare providers. This perspective is required because cost consequences of this study intervention will extend beyond the domain of healthcare. To conduct an economic analysis, the direct and indirect healthcare costs of the intervention and the participants will be acquired from the participant's cost diaries completed in the study. The economic analyses will include the following:

1) A cost-effectiveness within-study analysis will be conducted. Incremental cost-effectiveness ratios (ICER) will be calculated for the incremental increase in time and frequency domain parameter in the HRV-F group compared with the Control group.

2) A cost-utility analysis to determine incremental cost per quality-adjusted life-year (QALY) gained. The Australian valuation of the EQ-5D will be used to calculate utility weights on a $0-1$ scale where 1 represents very good health and 0 represents death. QALYs will be determined by multiplying utility by duration.

Resources will be valued using standard economic evaluation guidelines. Discounting will be set as $5 \%$ into the model.

\section{Excluded medications and treatments}

To maintain data validity and ensure participant safety, all participants will remain on their prescribed medications as well as any commonly used assistive technologies that will not interfere with measures such as HRV or EEG. Use of medications will be monitored via a selfreport assessment at each time point. Some medications may influence HRV, such as anti-hypertensives and antidepressants. However, $\beta$-blockers have been shown to change HRV substantially [66], participants taking $\beta$ blockers will be excluded. In a recent study [26], it was concluded that the majority of medications taken by adults with SCI were not associated with any abnormal influences on HRV data. This included cardiac medications such as ace inhibitors, angiotensin II, anti- platelet, calcium channel blockers, muscle relaxants and anticonvulsants. Even though it is expected that these medications will have some influence on HRV measures, randomization should control any such affects. However, if the data suggests that medication usage is not balanced across the RCT arms, then medication usage will be adjusted for in the statistical analysis to limit potential confounding.

\section{Control of bias}

A number of strategies will be used to manage bias. Intention-to-treat analysis involves all randomized participants being included in the statistical analysis according to the group they were originally assigned, regardless of whether they received all or any of the treatment. Intention-to-treat analysis helps to provide an unbiased estimate of the effectiveness of assignment to the HRV-F intervention. Selection bias will be controlled using concealed randomization and multivariable analysis adjusted for potential confounders such as education and age, as well as stratifying for level of lesion and sex. Furthermore, only adults with a chronic SCI will be recruited into the study and inclusion criteria will be strictly adhered to, so as to reduce confounding of data. Performance bias will be minimized by appropriate blinding to group assignment. Attrition bias addressed by using statistical analyses like linear mixed model analysis with repeated measures, as well as regular follow-up/ communication with participants in both groups, the length of the assessments will be kept at a minimum, small financial participation incentives will be used and 
final results of the study will be made available to all participants.

\section{Adverse events}

HRV-F is a low risk self-regulation intervention. It is unlikely to result in adverse events caused by the intervention or the devices used in the study. To monitor possible adverse events the following will occur: (a) A detailed record of all related and unrelated adverse events will be documented and kept in a locked cabinet or online in a password protected file. (b) The impact of each event on participant safety or on trial conduct will be clarified. (c) Assess and categorise the safety reports received from investigators. (d) Report all suspected unexpected serious adverse reactions. (v) Report to the sponsor (The University of Sydney) within 24-h of becoming aware of any serious adverse events. (vi) Report to the sponsor all safety critical events and any additional requested information relating to reported deaths.

\section{Power analyses}

For the primary outcome measures, assuming an $\alpha=$ 0.05 , a small to moderate effect size of 0.3 (based on results from studies in other conditions), with 2 groups (HRV-F and control) of 50 in each group and four assessment periods, estimated statistical power is 0.99 [67]. To allow for reduced participant numbers due to loss to follow-up, sickness, death, or unwillingness to continue, we will recruit up to 120 participants.

\section{Statistical methods}

Descriptive statistics will be generated for all relevant primary and secondary outcomes at all time-points. Linear mixed models for repeated measures analyses will be used to determine the effectiveness of the HRV-F intervention in autonomic function/HRV and cerebral blood circulation in basal cerebral arteries blood flow. Secondary analyses will determine whether improvements occur in factors such as mental health, cognitive function and sleep associated with the HRV-F intervention. All analyses will be adjusted for factors suspected to confound results, such as education and relevant baseline scores. Latent class mixture modelling will be used to determine trajectories for primary and selected secondary measures like anxiety, depressive mood and quality of life. Missing data will be managed using the linear mixed models, for example, through fitting maximum likelihood estimations generating asymptotically unbiased parameter estimates [68]. Data analyses will be performed using packages such as Statistical Package for the Social Sciences (SPSS version 27, SPSS inc., Chicago, Illinois, USA).
For the implementation study, in which qualitative analyses will also be used, the data will be analysed using valid qualitative packages. Grounded theory, an inductive technique of interpreting the recorded data about the perceptions of the HRV-F and its translation, will be the basis for reaching conclusions about how best to implement and translate the treatment.

For cost-effectiveness, descriptive statistics will be calculated to determine costs and QALYs. The robustness of the costing data will be examined through sensitivity analyses. As in standard economic assessments, costs acquired in this study will likely be skewed, so nonparametric bootstrap methods will potentially be used for testing hypotheses tests and interval estimation. A threshold ICER for Australia will be used to assess value for money.

The ICER will be calculated as per equation (eq. 1) below, that is, the incremental cost per increase in time and frequency domain parameters and as incremental cost per QALY gained in the intervention compared to the controls, in the cost-utility analysis.

$$
\text { ICER }=\frac{\text { Cost of intervention-Cost of control }}{\text { Outcome of intervention-Outcome of control }}
$$

Non-parametric bootstrapping will be used to estimate uncertainty around the ICER and results will be presented on an incremental cost-effectiveness plane. We will use 2021 AU\$ as the currency and will consequently derive ICERs and cost-effectiveness acceptability curves.

\section{Data monitoring committee}

To guarantee the participants' safety, an independent Data Monitoring Committee will meet each year of the study to review and evaluate the study data regarding the safety of the HRV-F intervention, the integrity of the data, appropriate study conduct and progress. The committee will consist of three experts who have no conflicts of interests with any of the researchers.

\section{Ethical approval and registration}

The trial received human research ethics approval (2020/ETH02554) on the 2nd December 2020 from the Northern Sydney Local Health District Human Research Ethics Committee. The trial has been registered at Australian and New Zealand Clinical Trial Registry (ACTRN 12621000870853.aspx) on 6 July 2021. SPIRIT guidelines have been followed for this protocol paper.

\section{Discussion}

Dysfunctional autonomic nervous system and brain activity is associated with significant health problems such 
as cardiovascular disorder, mental health disorder, sleep disorder, fatigue and cognitive impairment [11, 15-17], and is associated with potentially life threatening conditions in SCI [4, 11, 26, 69]. Treatments for improving autonomic function after SCI are limited so it is timely to establish whether a neuro-cardiac self-regulation therapy like HRV-F can result in improved autonomic and brain function post-SCI. Effectiveness will be assessed by primary outcomes like HRV and basal artery blood flow. It will also be important to establish whether HRV-F is associated with improved secondary conditions such as brain activity, fatigue, sleep, pain, cognitive capacity and mental health. If HRV-F is shown to be an effective intervention for those with chronic SCI, it is crucial that this treatment is translated into the community where it can be implemented by health professionals. Consequently, this study will also conduct an implementation mixed methods study to develop guidelines that can be adopted and translated into the community with the ultimate intention of improving the health of individuals with chronic SCI.

\begin{abstract}
Abbreviations
ANS: Autonomic nervous system; SCl: Spinal cord injury; AD: Autonomic dysreflexia; BP: Blood pressure; BPV: Blood pressure variability;

fTCD: Functional transcranial doppler sonography; RCT: Randomized controlled trial; BPM: Breaths per minute; SDNN: Standard deviation of the average NN intervals; RSA: Respiratory sinus arrhythmia;

EEG: Electroencephalography; EOG: Electrooculography; NIRS: Near infrared spectroscopy; NUCOG: Neuropsychiatry Unit Cognitive Assessment Tool; HRV: Heart rate variability; HRV-F: Neuro-cardiac self-regulation therapy; HF: High frequency; LF: Low frequency; ADFSCl: Autonomic Dysfunction Following Spinal Cord Injury; ISCoS: International Spinal Cord Society; GAD7: Generalised Anxiety Disorder-7; PHQ-9: Patient Health Questionnaire-9; PCL-5-SF: Post-traumatic Stress Disorder Checklist for the Diagnostic and Statistical Manual of Mental Disorders Version V Short-Form; SSQ-6: Social Support Questionnaire Short Form; PCS: Pain Catastrophizing Scale; FSS: Fatigue Severity Scale; PSQI: The Pittsburgh Sleep Quality Index; BQ: Berlin Questionnaire; SCI-SCS: Spinal Cord Injury Secondary Conditions Scale; WHO ASSIST: World Health Organisation Alcohol, Smoking, and Substance Involvement Screening Test; Brief COPE: Brief Coping Orientation to Problems Experienced; MSES: Moorong Self-Efficacy Scale; CDRISC2: Connor-Davidson Resilience Scale; ADAPSS: Appraisals of DisAbility: Primary and Secondary Scale Short-Form; EQ-5D-5L: EUROQOL version 5D-5L; WHODAS-2: World Health Organization Disability Assessment Schedule; REDCap: Research Electronic Data Capture; SPSS: Statistical Package for the Social Sciences; TDF: Theoretical Domains Framework; SSCIS: State Spinal Cord Service
\end{abstract}

\section{Acknowledgments}

We acknowledge the NSW Ministry of Health and The University of Sydney for funding the study.

\section{Authors' contributions}

AC drafted the protocol and substantively contributed to the design of the study. JM, IP, MA, IC, BG, GMD, YT, AK1, JB, RM, AK2, SG all substantively contributed to the design of the study and the revision of the protocol. CM, DR and JS contributed to the writing and revision of the protocol. AK1 provided statistical advice which was central to the study design. We declare that all authors read and approved the final manuscript that was submitted for publication.

\section{Funding}

The study was competitively funded by the NSW Ministry of Health, Australia and The University of Sydney, Australia. The NSW Ministry of Health peer reviewed the application, seeking international anonymous reviews on the research protocol from international experts which the investigators were able to address. Otherwise, the funding source had no role in the design of this study and will not have any role during its execution, analyses, interpretation of the data, or decision to submit results.

\section{Availability of data and materials}

All chief investigators will have access to the final dataset. All datasets used and/or analyzed during the current study are available from the corresponding author on reasonable request.

\section{Declarations}

\section{Ethics approval and consent to participate}

The Northern Sydney Local Health District (NSLHD) Human Research Ethics Committee approved the study on the 2nd December, 2020 (2020/ ETH02554) and Site Specific Authorization for each of the study sites has been granted. Notification of any protocol modifications will be made to the NSLHD Ethics Committee. The principals of the Declaration of Helsinki and Good Clinical Practice guidelines will be adhered to. Participants in this study will provide written informed consent. Provisions for withdrawal from the study at any stage will be incorporated into the consent process and participants will be explicitly informed verbally and in writing that decisions regarding non-participation/participation will not affect their current or future relationships with treating hospitals or the Principal Investigators.

\section{Consent for publication}

Not applicable.

\section{Competing interests}

IP, MA, CM, AK, JS receive part-time salaries from funding received for this study.

\section{Author details}

${ }^{1}$ John Walsh Centre Rehabilitation Research, Northern Sydney Local Health District, The Kolling Institute, Northern Clinical School, Faculty of Medicine and Health, The University of Sydney, St Leonards, NSW 2065, Australia. ${ }^{2}$ Macquarie University Hearing (MU Hearing), Macquarie University, North Ryde, NSW 2113, Australia. ${ }^{3}$ Exercise and Sports Sciences, Faculty of Medicine and Health, The University of Sydney, Sydney, NSW, Australia. ${ }^{4}$ ICORD, Faculty of Medicine, University of British Columbia, Vancouver, British Columbia V5Z $1 \mathrm{M9}$, Canada. ${ }^{5}$ Centre for Healthcare Resilience and Implementation Science, Australian Institute of Health Innovation, Health Systems Research, Macquarie University, North Ryde, NSW 2113, Australia. ${ }^{6}$ School of Psychology, Faculty of Science, University of New South Wales, Kensington, NSW, Australia.

Received: 22 July 2021 Accepted: 13 August 2021

Published online: 26 August 2021

\section{References}

1. Alizadeh A, Dyck SM, Karimi-Abdolrezaee S. Traumatic spinal cord injury: an overview of pathophysiology, models and acute injury mechanisms. Front Neurol. 2019;10:282. https://doi.org/10.3389/fneur.2019.00282.

2. Grassner L, Marschallinger J, Dünser MW, Novak HF, Zerbs A, Aigner L, et al. Nontraumatic spinal cord injury at the neurological intensive care unit: spectrum, causes of admission and predictors of mortality. Ther Adv Neurol Disord. 2016;9(2):85-94. https://doi.org/10.1177/1756285615621687.

3. Giannoccaro MP, Moghadam KK, Pizza F, Boriani S, Maraldi NM, Avoni P, et al. Sleep disorders in patients with spinal cord injury. Sleep Med Rev. 2013;17(6):399-409. https://doi.org/10.1016/j.smrv.2012.12.005.

4. Craig A, Rodrigues D, Tran Y, Guest R, Middleton J. Daytime sleepiness and its relationships to fatigue and autonomic dysfunction in adults with spinal cord injury. J Psychosom Res. 2018;112:90-8. https://doi.org/10.1016/j. jpsychores.2018.07.007.

5. Craig A, Nicholson Perry K, Guest R, Tran Y, Dezarnaulds A, Hales A, et al. Prospective study of the occurrence of psychological disorders and comorbidities after spinal cord injury. Arch Phys Med Rehabil. 2015;96(8): 1426-34. https://doi.org/10.1016/j.apmr.2015.02.027.

6. Craig A, Guest R, Tran Y, Nicholson Perry K, Middleton J. Pain catastrophizing and negative mood states following spinal cord injury: 
transitioning from inpatient rehabilitation into the community. J Pain. 2017; 18(7):800-10. https://doi.org/10.1016/j.jpain.2017.02.431.

7. Siddall PJ, McClelland JM, Rutkowski SB, Cousins MJ. A longitudinal study of the prevalence and characteristics of pain in the first 5 years following spinal cord injury. Pain. 2003;103(3):249-57. https://doi.org/10.1016/S0304-3 959(02)00452-9.

8. Caig A, Tran Y, Wijesuriya N, Middleton J. Fatigue and tiredness in people with spinal cord injury. J Psychosom Res. 2012;73:205-10.

9. Sachdeva R, Gao F, Chan CCH, Krassioukov AV. Cognitive function after spinal cord injury: a systematic review. Neurology. 2018;91(13):611-21. https://doi.org/10.1212/WNL.0000000000006244.

10. Sandalic D, Craig A, Arora M, Pozzato I, Simpson G, Gopinath B, et al. A prospective cohort study investigating contributors to mild cognitive impairment in adults with spinal cord injury: study protocol. BMC Neurol. 2020;20(1):341.

11. Krassioukov A. Autonomic function following cervical spinal cord injury. Respir Physiol Neurobiol. 2009;169(2):157-64. https://doi.org/10.1016/j.resp.2 009.08.003.

12. Phillips AA, Squair JW, Sayenko DG, Edgerton VR, Gerasimenko Y, Krassioukov AV. An autonomic neuroprosthesis: noninvasive electrical spinal cord stimulation restores autonomic cardiovascular function in individuals with spinal cord injury. J Neurotrauma. 2018;35(3):446-51. https://doi.org/1 0.1089/neu.2017.5082.

13. Darrow D, Balser D, Netoff TI, Krassioukov A, Phillips A, Parr A, et al. Epidural spinal cord stimulation facilitates immediate restoration of dormant motor and autonomic supraspinal pathways after chronic neurologically complete spinal cord injury. J Neurotrauma. 2019;36(15):2325-36. https://doi.org/10.1 089/neu.2018.6006.

14. Lehrer P, Vaschillo B, Zucker T, Graves J, Katsamanis M, Aviles M, et al. Protocol for heart rate variability biofeedback training. Biofeedback. 2013; 41(3):98-109. https://doi.org/10.5298/1081-5937-41.3.08.

15. GoessI VC, Curtiss JE, Hofmann SG. The effect of heart rate variability biofeedback training on stress and anxiety: a meta-analysis. Psychol Med. 2017;47(15):2578-86. https://doi.org/10.1017/S0033291717001003.

16. Lehrer P, Vaschillo E, Lu SE, Eckberg D, Vaschillo B, Scardella A, et al. Heart rate variability biofeedback: effects of age on heart rate variability, baroreflex gain, and asthma. Chest. 2006;129(2):278-84. https://doi.org/10.1378/ chest.129.2.278

17. Hassett AL, Radvanski DC, Vaschillo EG, Vaschillo B, Sigal LH, Karavidas MK, et al. A pilot study of the efficacy of heart rate variability (HRV) biofeedback in patients with fibromyalgia. App Psychophysiol Biofeedback. 2007;32(1):110. https://doi.org/10.1007/s10484-006-9028-0.

18. Del Pozo JM, Gevirtz RN, Scher B, Guarneri E. Biofeedback treatment increases heart rate variability in patients with known coronary artery disease. Am Heart J. 2004;147:e11.

19. Lehrer PM, Gevirtz R. Heart rate variability biofeedback: how and why does it work? Front Psychol. 2014:5:756

20. Park $G$, Thayer JF. From the heart to the mind: cardiac vagal tone modulates top-down and bottom-up visual perception and attention to emotional stimuli. Front Psychol. 2014;5:278.

21. Oikawa O, Malinovsky I, Kotay A, Karavidas MK, Sudo K, Tashiro K, et al. Heart rate variability biofeedback: new directions in collaborative medical and related healthcare research. Japanese J Biofeedback Res. 2007;34:17-21.

22. Sitaram R, Ros T, Stoeckel L, Haller S, Scharnowski F, Lewis-Peacock J, et al. Closed-loop brain training: the science of neurofeedback. Nat Rev Neurosci. 2017;18(2):86-100. https://doi.org/10.1038/nrn.2016.164.

23. Sachdeva R, Nightingale TE, Krassioukov AV. The blood pressure pendulum following spinal cord injury: implications for vascular cognitive impairment. Int J Molecular Sci. 2019:20(10):2464. https://doi.org/10.3390/ijms20102464.

24. Claydon VE, Krassioukov AV. Orthostatic hypotension and autonomic pathways after spinal cord injury. J Neurotrauma. 2006;23(12):1713-25 https://doi.org/10.1089/neu.2006.23.1713.

25. Biering-Sørensen F, Biering-Sørensen T, Liu N, Malmqvist L, Wecht JM, Krassioukov A. Alterations in cardiac autonomic control in spinal cord injury. Autonom Neurosci. 2018;209:4-18. https://doi.org/10.1016/j.autneu.2017.02 004

26. Rodrigues D, Tran Y, Guest R, Middleton J, Craig A. Influence of neurological lesion level on heart rate variability and fatigue in adults with spinal cord injury. Spinal Cord. 2016;54(4):292-7. https://doi.org/10.1038/sc.2015.174.

27. Craig A, Tran Y, Guest R, Middleton J. Excessive daytime sleepiness in adults with spinal cord injury and associations with pain catastrophizing and pain intensity. Spinal Cord. 2020;58(7):831-9. https://doi.org/10.1038/s41393-0200425-7.

28. Bazanova OM, Balioz NV, Muravleva KB, Skoraya MV. Effect of voluntary EEG a power increase training on heart rate variability. Hum Physiol. 2013;39(1): 86-97. https://doi.org/10.1134/S0362119712060035.

29. Prinsloo GE, Rauch HL, Karpul D, Derman WE. The effect of a single session of short duration heart rate variability biofeedback on EEG: a pilot study. App Psychophysiol Biofeedback. 2013;38(1):45-56. https://doi.org/10.1007/s1 0484-012-9207-0.

30. Boord P, Siddall PJ, Tran Y, Herbert D, Middleton J, Craig A. Electroencephalographic slowing and reduced reactivity in neuropathic pain following spinal cord injury. Spinal Cord. 2008;46(2):118-23. https://doi. org/10.1038/sj.sc.3102077.

31. Tran $Y$, Boord $P$, Middleton J, Craig A. Levels of brain activity $(8-13 \mathrm{~Hz})$ in persons with spinal cord injury. Spinal Cord. 2004;42(2):73-9. https://doi. org/10.1038/sj.sc.3101543.

32. Beissner F, Meissner K, Bär KJ, Napadow V. The autonomic brain: an activation likelihood estimation meta-analysis for central processing of autonomic function. J Neurosci. 2013;33(25):10503-11. https://doi.org/10.1 523/JNEUROSCI.1103-13.2013.

33. Phillips AA, Ainslie PN, Krassioukov AV, Warburton DE. Regulation of cerebral blood flow after spinal cord injury. J Neurotrauma. 2013;30(18):1551-63. https://doi.org/10.1089/neu.2013.2972.

34. Nolan RP, Kamath MV, Floras JS, Stanley J, Pang C, Picton P, et al. Heart rate variability biofeedback as a behavioral neurocardiac intervention to enhance vagal heart rate control. Am Heart J. 2005;149:1137-e1.

35. Thayer JF, Lane RD. A model of neurovisceral integration in emotion regulation and dysregulation. J Affective Dis. 2000;61(3):201-16. https://doi. org/10.1016/S0165-0327(00)00338-4.

36. Harris PA, Taylor R, Thielke R, Payne J, Gonzalez N, Conde JG. Research electronic data capture (REDCap)-a metadata-driven methodology and workflow process for providing translational research informatics support. J Biomed Inform. 2009;42(2):377-81. https://doi.org/10.1016/j.jbi.2008.08.010.

37. Shaffer F, Meehan ZM. A practical guide to resonance frequency assessment for heart rate variability biofeedback. Front Neurosci. 2020;14:570400. https://doi.org/10.3389/fnins.2020.570400.

38. Michie S, Johnston M, Abraham C, Lawton R, Parker D, Walker A. Making psychological theory useful for implementing evidence-based practice: consensus approach. BMJ Qual Safety. 2005;14(1):26-33. https://doi.org/1 $0.1136 /$ ashc.2004.011155

39. Clay-Williams R, Nosrati H, Cunningham FC, Hillman K, Braithwaite J. Do large-scale hospital-and system-wide interventions improve patient outcomes: a systematic review. BMC Health Serv Res. 2014;14(1):369. https:// doi.org/10.1186/1472-6963-14-369.

40. Tran Y, Wijesuriya N, Tarvainen M, Karjalainen P, Craig A. The relationship between spectral changes in HRV and fatigue. J Psychophysiol. 2009;23(3): 143-51. https://doi.org/10.1027/0269-8803.23.3.143.

41. Duschek S, Schandry R. Functional transcranial Doppler sonography as a tool in psychophysiological research. Psychophysiology. 2003;40(3):436-54 https://doi.org/10.1111/1469-8986.00046.

42. Kushki A, Fairley J, Merja S, King G, Chau T. Comparison of blood volume pulse and skin conductance responses to mental and affective stimuli at different anatomical sites. Physiol Measure. 2011;32(10):1529-39. https://doi. org/10.1088/0967-3334/32/10/002.

43. Chen WL, Wagner J, Heugel N, Sugar J, Lee YW, Conant L, et al. Functional near-infrared spectroscopy and its clinical application in the field of neuroscience: advances and future directions. Front Neurosci. 2020;14:724. https://doi.org/10.3389/fnins.2020.00724.

44. Hubli M, Gee CM, Krassioukov AV. Refined assessment of blood pressure instability after spinal cord injury. Am J Hypertension. 2015;28(2):173-81. https://doi.org/10.1093/ajh/hpu122.

45. Kalpakjian CZ, Scelza WM, Forchheimer MB, Toussaint LL. Preliminary reliability and validity of a spinal cord injury secondary conditions scale. J Spinal Cord Med. 2007;30(2):131-9. https://doi.org/10.1080/10790268.2 007.11753924

46. Walterfang $M$, Siu R, Velakoulis D. The NUCOG: Validity and reliability of a brief cognitive screening tool in neuropsychiatric patients. Australian NZ J Psychiatry 2006:40:995-1002. https://doi.org/10.1080/j.1440-1614.2006.01923. x.

47. Craig A, Guest R, Tran Y, Middleton J. Cognitive impairment and mood states after spinal cord injury. J Neurotrauma. 2016;34:1156-63. 
48. Spitzer RL, Kroenke K, Williams JBW, Löwe B. A brief measure for assessing generalized anxiety disorder: the GAD-7. Arch Intern Med. 2006;166(10): 1092-7. https://doi.org/10.1001/archinte.166.10.1092.

49. Kroenke K, Spitzer RL, Williams JB. The PHQ-9: validity of a brief depression severity measure. J Gen Intern Med. 2001;16(9):606-13. https://doi.org/10.1 046/j.1525-1497.2001.016009606.x

50. Widerström-Noga E, Biering-Sørensen F, Bryce T, Cardenas DD, Finnerup $N B$, Jensen MP, et al. The international spinal cord injury pain basic data set. Spinal Cord. 2008;46(12):818-23. https://doi.org/10.1038/sc.2008. 64.

51. Forchheimer MB, Richards JS, Chiodo AE, Bryce TN, Dyson-Hudson TA. Cut point determination in the measurement of pain and its relationship to psychosocial and functional measures after traumatic spinal cord injury: a retrospective model spinal cord injury system analysis. Arch Phys Med Rehabil. 2011;92(3):419-24. https://doi.org/10.1 016/j.apmr.2010.08.029.

52. Krupp LB, Larocca NG, Muir-Nash J, Steinberg AD. The fatigue severity scale; application to patients with multiple sclerosis and systemic lupus erythematosus. Arch Neurol. 1989;46:1121-3.

53. Buysse DJ, Reynolds CF, Monk TH, Berman SR, Kupfer DJ. The Pittsburgh sleep quality index: a new instrument for psychiatric practice and research. Psychiatry Res. 1989;28(2):193-213. https://doi.org/10.1016/0165-1781 (89)90047-4.

54. Sullivan MJL, Bishop SR, Pivik J. The pain catastrophizing scale: development and validation. Psychological Assess. 1995;7(4):524-32. https://doi.org/10.103 7/1040-3590.7.4.524.

55. Vaishnavi S, Connor K, Davidson JRT. An abbreviated version of the ConnorDavidson resilience scale (CD-RISC), the CD-RISC2: psychometric properties and applications in psychopharmacological trials. Psychiatry Res. 2007;152(23):293-7. https://doi.org/10.1016/j.psychres.2007.01.006

56. Herdman M, Gudex C, Lloyd A, Janssen MF, Kind P, Parkin D, et al. Development and preliminary testing of the new five-level version of EQ-5D (EQ-5D-5L). Quality Life Res. 2011;20(10):1727-36. https://doi.org/10.1007/ s11136-011-9903-X.

57. Garin O, Ayuso-Mateos JL, Almansa J, Nieto M, Chatterji S, Vilagut G, et al. Validation of the "World Health Organization disability assessment schedule, WHODAS-2" in patients with chronic diseases. Health Quality Life Out. 2010; 8:1-5.

58. Zuromski KL, Ustun B, Hwang I, Keane TM, Marx BP, Stein MB, et al. Developing an optimal short-form of the PTSD checklist for DSM-5 (PCL-5). Depress Anxiety. 2019;36(9):790-800. https://doi.org/10.1002/da.22942.

59. Carver CS. You want to measure coping but your protocol' too long: consider the brief cope. Int J Behav Med. 1997;4(1):92-100. https://doi.org/1 0.1207/s15327558ijbm0401_6.

60. Sarason IG, Sarason BR, Shearin EN, Pierce GR. A brief measure of social support: practical and theoretical implications. J Social Personal Relationships. 1987;4(4):497-510. https://doi.org/10.1177/0265407587044007.

61. Middleton J, Tran Y, Lo C, Craig A. Re-examining the validity and dimensionality of the Moorong self-efficacy scale: improving its clinical utility. Arch Phys Med Rehabil. 2016;97(12):2130-6. https://doi.org/10.1016/j.a pmr.2016.05.027.

62. McDonald SD, Goldberg-Looney LD, Mickens MN, Ellwood MS, Mutchler BJ, Perrin PB. Appraisals of DisAbility primary and secondary scale-short form (ADAPSS-Sf): psychometrics and association with mental health among U.S. military veterans with spinal cord injury. Rehabil Psychol. 2018;63(3):372-82. https://doi.org/10.1037/rep0000230.

63. Netzer NC, Stoohs RA, Netzer CM, Clark K, Strohl KP. Using the Berlin questionnaire to identify patients at risk for the sleep apnea syndrome. Ann Int Med. 1999;131(7):485-91. https://doi.org/10.7326/0003-4819-131-7-19991 0050-00002.

64. Humeniuk R, Henry-Edwards S, Ali R, Poznyak V, Monteiro MG, World $\mathrm{HO}$. The alcohol, smoking and substance involvement screening test (ASSIST): manual for use in primary care. Geneva: World Health Organization; 2010

65. Group WA. The alcohol, smoking and substance involvement screening test (ASSIST): development, reliability and feasibility. Addiction. 2002;97(9):118394. https://doi.org/10.1046/j.1360-0443.2002.00185.x.

66. Rabbia F, Silke B, Carra R, Milan A, Del Colle S, Pugni C, et al. Heart rate variability and baroreflex sensitivity during fosinopril, irbesartan and atenolol therapy in hypertension. Clin Drug Invest. 2004:24:661-59.

67. Faul F. G* Power Version 3.1. 9.2. Kiel: Universitat Kiel, Germany; 2014.
68. Little RJ, Raghunathan T. On summary measures analysis of the linear mixed effects model for repeated measures when data are not missing completely at random. Statistics Med. 1999;18(17-18):2465-78. https://doi.org/10.1002/ (SICI)1097-0258(19990915/30)18:17/18<2465::AID-SIM269>3.0.CO;2-2.

69. Wan D, Krassioukov AV. Life-threatening outcomes associated with autonomic dysreflexia: a clinical review. J Spinal Cord Med. 2014;37(1):2-10. https://doi.org/10.1179/2045772313Y.0000000098.

\section{Publisher's Note}

Springer Nature remains neutral with regard to jurisdictional claims in published maps and institutional affiliations.
Ready to submit your research? Choose BMC and benefit from:

- fast, convenient online submission

- thorough peer review by experienced researchers in your field

- rapid publication on acceptance

- support for research data, including large and complex data types

- gold Open Access which fosters wider collaboration and increased citations

- maximum visibility for your research: over $100 \mathrm{M}$ website views per year

At $\mathrm{BMC}$, research is always in progress.

Learn more biomedcentral.com/submissions 